COMMENT. Traumatic brain injury (TBI) is known to carry an increased risk of epilepsy, but factors that modify the incidence of epilepsy are not well defined. In a previous population-based study in Olmsted County, Minnesota, involving 4541 children and adults, the overall standardized incidence ratio for post-traumatic epilepsy was 3.1. For patients who had sustained a mild injury (loss of consciousness or amnesia for $<30 \mathrm{~min}$ ), the incidence ratio was 1.5, with no increase after 5 years; following moderate injury (loss of consciousness $30 \mathrm{~min}$ to $24 \mathrm{hrs}$ or skull fracture) it was 2.9; and after severe injury (loss of consciousness or amnesia $>24 \mathrm{hrs,}$, subdural hematoma, or brain contusion) the incidence ratio was 17.0 (Annegers JF et al. N Engl J Med 1998;338:20-24). Patients in the Minnesota study with an increased incidence of epilepsy after 5 years had a history of severe brain injury and were age 65 yrs or older. In the Danish study, the risk of epilepsy was high for more than 10 years even after mild brain injury (concussion) in younger patients. Family history of epilepsy and mild brain injury independently contributed to the risk of posttraumatic epilepsy in children and young adults.

Prophylactic phenytoin does not reduce the incidence of early or late seizures following brain injury in children (Teasell R et al. Brain Inj 2007;21:201-214). Despite disappointing results of trials of prophylactic antiepileptic medication in head injury patients (Temkin NR. Epilepsia 2001;42:515-524), the Danish authors suggest their data warrant further study of newer agents in high risk patients. The evidence suggests that prevention of injury offers greater promise of success than prophylactic medication in reducing the prevalence of post-traumatic epilepsy.

\title{
RELATION OF AGE AT INSULT TO OUTCOME OF BRAIN INJURY
}

Cognitive and behavioral outcomes for children who sustain early brain insult (EBI) were evaluated in relation to age at insult in a study at Royal Children's Hospital, Victoria, Australia. Grouped according to age at time of focal brain insult, 36 sustained congenital (first-second trimester) injuries, 33 perinatal (third trimester to 1 month post-natal), 23 were in infancy ( 2 months to 2 years after birth), 19 preschool (3 to 6 years), 31 mid-childhood (79 years), and 19 late childhood (after age 10 years). Children were tested for intelligence, academic ability, executive function, and behavior. Children with EBI were at increased risk for impairment in all domains of cognition and behavior, with mean scores falling 1SD below expectations. Low scores in arithmetic were especially common, occurring in $63 \%$ of EBI children. EBI before 2 years resulted in global cognitive deficits, whereas injury sustained after 2 years of age was followed by near normal cognitive development. In contrast, behavior was worse in older children with EBI from 7 to 9 years compared to those sustaining injury from 3 to 6 years. The age at the time of brain insult is important in predicting risk of cognitive and behavioral outcomes in children with EBI, but patterns of vulnerability differ with respect to age at insult. (Anderson V, Spencer-Smith M, Leventer R, et al. Childhood brain insult: can age at insult help us predict outcome? Brain Jan 2009;132:45-56). (Respond: Vicki Anderson, Department of Psychology, Royal Children's Hospital, Flemington Road, Parkville, Victoria, 3052, Australia. E-mail: vicki.anderson@,rch.org.au).

COMMENT. Children who sustain an early brain insult are at increased risk of developing impairments of cognition and behavior. Injury in the pre- or perinatal period or in 
early childhood results in cognitive deficits whereas injury in later childhood is more likely to result in behavioral problems. The findings indicate an increased vulnerability of the young brain and a lack of evidence to support theories of brain plasticity. Children with a history of brain injury before, at birth or before 2 years of age are at risk of persistent impairments of learning that require early interventional therapy.

\section{HEADACHE DISORDERS}

\section{MIGRAINE AND SUICIDAL IDEATION IN ADOLESCENTS}

The relationship between migraine and suicidal ideation (self-reported thoughts of suicide-related behaviors) in a sample of young adolescents was determined in a study at Taipei Veterans General Hospital, Taipei, Taiwan. Students in three middle schools completed a validated headache questionnaire, the Adolescent Depression Inventory (ADI), and the Pediatric Migraine Disability Assessment questionnaire. The questionnaires assessed the headache profile during the past 3 months and symptoms of depression in the past month. "I think about killing myself" was the indicator of self-reported suicidal ideation. Suicidal ideation in the past month was reported in $8.5 \%$ of 3,963 adolescents (2040 male and 1923 female; mean age $14.0+/-0.9$ years) who completed the study. According to the International Classification of Headache Disorders, 928 subjects $(23.4 \%)$ were diagnosed with migraine; $138(3.5 \%)$ had migraine with aura, $346(8.7 \%)$ migraine without aura, and $444(11.2 \%)$ with probable migraine. The frequency of suicidal ideation was $6.2 \%$ in nonmigraine subjects compared to $16.1 \%$ in subjects with migraine $(\mathrm{p}<0.001)$, and $23.9 \%$ in subjects with migraine with aura $(\mathrm{p}<0.001)$. Subjects with suicidal ideation had a higher frequency of headache and headache-related disability. After controlling for depression and sociodemographic factors, the association of migraine and suicidal ideation occurred only for migraine with aura $(p=0.025)$ and high frequency headache $(>7$ days/month; $p=0.013)$, but not for migraine without aura, probable migraine or for migraine disability score. (Wang S-J, Fuh J-L, Juang K-D, Lu S-R. Migraine and suicidal ideation in adolescents aged 13 to 15 years. Neurology March 31, 2009;72(13):1146-1152). (Respond and reprints: Dr Shuu-Jiun Wang, The Neurological Institute, Taipei Veterans General Hospital, Taipei, 112, Taiwan. Email: sjwang@,vghtpe.gov.tw).

COMMENT. One in four young adolescents with migraine with aura and one in four with frequent headaches ( $>7$ days/month) report suicidal ideation. The frequency of suicidal ideation is one in 2.5 for subjects with both risk factors. The association of migraine with aura and suicidal ideation is independent of depression and pain. Alterations of the serotonergic system have been demonstrated in subjects with both migraine with aura and suicide. (Post RM et al. Neurology 1994;44 (suppl 7):537-547). The current authors have previously shown that adolescents with chronic daily headaches $(>15$ days/month) are at increased risk of suicide. (Wang SJ et al. Neurology 2007;68:1468-1473). The influence of prophylactic medications such as antiepileptics and antidepressants was not evaluated in this study, but chronic prophylactic migraine therapy was used infrequently in this age group. The study demonstrates the importance of evaluation for risk factors of suicidal thoughts in young adolescents with migraine with aura. 